\title{
Corela
}

Cognition, représentation, langage

13-1 | 2015

Vol. $13, \mathrm{n}^{\circ} 1$

\section{Récit, Discours Direct et Discours Indirect : passerelles référentielles en moyen français}

Estèle Dupuy

\section{OpenEdition}

Journals

Édition électronique

URL : http://journals.openedition.org/corela/3944

DOI : $10.4000 /$ corela.3944

ISSN : 1638-573X

Éditeur

Cercle linguistique du Centre et de l'Ouest - CerLICO

Référence électronique

Estèle Dupuy, «Récit, Discours Direct et Discours Indirect : passerelles référentielles en moyen français », Corela [En ligne], 13-1 | 2015, mis en ligne le 30 juin 2015, consulté le 01 mai 2019. URL : http://journals.openedition.org/corela/3944; DOI : 10.4000/corela.3944

Ce document a été généré automatiquement le 1 mai 2019.

\section{(c) (i) (2)(2)}

Corela - cognition, représentation, langage est mis à disposition selon les termes de la licence Creative Commons Attribution - Pas d'Utilisation Commerciale - Partage dans les Mêmes Conditions 4.0 International. 


\title{
Récit, Discours Direct et Discours Indirect : passerelles référentielles en moyen français
}

\author{
Estèle Dupuy
}

1 Lorsque nous passons du récit au discours direct (désormais DD) ou au discours indirect (DI), un fil conducteur sous-jacent nous permet de savoir à tout moment de quoi il est question et qui est en cause dans l'acte locutoire comme dans la délocution. La chaine anaphorique est envisagée par nous comme la combinaison de l'anaphore et de la coréférence ${ }^{1}$. Elle réunit linéairement toutes les chaînes de références (Schnedecker, 1997 et Landragin \& Schnedecker, 2014) ayant une même entité comme référent. Plusieurs mécanismes assurent au sein de la châne anaphorique le maintien de la continuité référentielle. Ainsi, comme nous allons le montrer, les expressions anaphoriques et coréférentielles et leur choix-même sont porteurs d'indications concernant, certes le référent, mais aussi la continuité référentielle. En effet, à la reprise d'un référent, l'utilisation d'un nom, d'un pronom ou d'une forme zéro ${ }^{2}$ véhicule des informations quant à l'accessibilité du référent (Prince : 1981, Ehlich : 1982 et 1983, Kleiber : 1991). Entrent alors en jeu des mécanismes concernant la concurrence référentielle entre deux ou plusieurs référents, le maintien de ce référent dans un rôle valentiel constant ou non (actant 1 ou actant 2, par exemple), la position syntaxique des rôles valentiels. Ces mécanismes complémentaires, étroitement liés à la sémantique verbale et à la syntaxe propositionnelle, sous-tendent le choix de ces expressions. Se créé ainsi un filage référentiel qui assure la continuité référentielle (Dupuy-Parant, 2006).

Ces théories ont pu être vérifiées, à partir d'un corpus de moyen français (MF) constitué de trois textes ${ }^{3}$ en prose dont deux sont d'un genre proche (Chroniques et Mémoires) mais distants d'environ un siècle et le troisième relève d'un genre différent (proche de la nouvelle et présentant des référents résomptifs à l'inverse des deux autres textes) mais chronologiquement proche des Chroniques. Le choix de ces trois textes permet ainsi une comparaison triangulaire (de genre littéraire, diachronique et, dans une moindre mesure, 
dialectale ${ }^{4}$ qui offre la possibilité de distinguer ce qui relève d'une évolution diachronique et ce qui peut être plus spécifique à un genre.

3 Pour préciser le contexte de cette recherche, nous avons pu montrer dans des études antérieures ${ }^{5}$ que le choix des expressions anaphoriques réparties en trois catégories :

- les anaphores nominales répondant à deux catégories référentielles différentes : redéfinitions ${ }^{6}$ (anaphore lexicale infidèle ${ }^{7}$, pronom indéfini, déterminant démonstratif suivi d'un nom renommant, anaphore avec déterminant ledict) ${ }^{8}$ et réinitialisations (anaphore lexicale fidèle propre ou impropre sans ledict $)^{9}$;

- les anaphores pronominales réalisées par le pronom personnel, le pronom relatif, interrogatif et le pronom démonstratif seul ;

- les anaphores zéro pour lesquelles seule la désinence verbale (non ambigüe en MF) permet d'identifier la personne verbale,

4 n'est pas subjectif mais répond à une combinatoire de cinq règles syntactico-sémantiques commune aux trois milieux discursifs : récit, DD et DI.

5 Une règle de concurrence référentielle privilégie l'anaphore nominale lorsque les référents en présence sont de même nature (ex: animé humain), genre et nombre et induisent la même personne verbale. Si l'un de ces critères diffère, l'anaphore pronominale ou zéro suffisent.

Trois règles dites valentiello-référentielles allient la référenciation à la valence verbale en prenant en compte répartition des expressions anaphoriques sur les rôles valentiels des verbes successifs : l'une est une règle intra-distributionnelle ${ }^{10}$ et les deux autres sont des règles inter-distributionnelles; l'une concerne l'actant 1 , l'autre, l'actant $2^{11}$.

7 La cinquième règle dite syntactico-sémantique contraint les deux précédentes en fonction de la catégorie grammaticale dans laquelle entre la proposition accueillant la distribution valentielle du verbe ${ }^{12}$.

8 L'objectif, ici, n'est pas de présenter la validité de cette combinatoire par ailleurs attestée dans nos trois milieux discursifs (Dupuy, 2012) mais de présenter les spécificités de fonctionnement du DD et du DI liées à la spécificité de ces milieux. La particularité principale de ceux-ci est la dimension du locuteur.

9 Ainsi, en DD, la combinatoire s'applique-t-elle aux référents, thèmes de la conversation (induisant des $\mathrm{P} 3$ ou 6), et non aux locuteurs. Or les locuteurs ont un rôle à jouer dans le choix de certaines expressions anaphoriques...

$10 \mathrm{En} \mathrm{DI}^{13}$ la combinatoire est également appliquée pour les référents-thèmes. Les référentslocuteurs (induisant aussi une $\mathrm{P} 3$ ou 6) subissent un traitement anaphorique particulier qui ne suit aucune règle de la combinatoire. Ils y sont toujours repris par anaphore pronominale ${ }^{14}$ sans que cela génère d'ambiguïté référentielle.

11 Ainsi, en discours, certaines expressions anaphoriques ne sont pas explicables par la combinatoire telle qu'elle s'applique en récit et dans la majeure partie du DI et du DD. Pour comprendre et décrire ces différences, nous observerons l'impact en DD et DI de la présence de locuteurs et la manifestation d'une mémoire référentielle intra ou extraséquentielle ${ }^{15}$. De plus, nous formulerons des hypothèses et des analyses sur le degré de porosité référentielle entre les différents milieux discursifs (récit, DD et DI) afin de mettre au jour les spécificités de fonctionnement de la continuité référentielle au passage de l'un à l'autre et de soulever des hypothèses quant à l'existence de passerelles référentielles entre ces milieux. 
12 Notre présentation commencera par l'observation et l'analyse des relations référentielles au sein d'une même séquence dialogale ${ }^{16}$ (relations intra-séquentielles) en ciblant les indices de mémoire intra-séquentielle (en DD et DI) et la dimension du locuteur pour le DD. Puis, elle abordera les indices de mémoire inter-séquentielle ${ }^{17}$ en se basant sur les relations référentielles qui peuvent être entretenues entre deux ou plusieurs séquences dialogales qui présentent un même thème de discussion et une communauté de locuteurs pour partie commune. Enfin, sera posée l'hypothèse de l'existence de passerelles référentielles entre le récit et le DD - le DI pouvant jouer un rôle de passeur - qui seraient à l'origine de la continuité référentielle entre les trois milieux discursifs.

\section{Relations intra-séquentielles}

13 Certaines expressions anaphoriques utilisées au sein d'une même séquence dialogale en DD comme en DI ne résultent pas de la combinatoire syntactico-sémantique mais sont à mettre en rapport avec la présence de locuteurs en milieu discursif. Partant, elles indiquent des opérations liées qui marquent d'une part, la présence d'une mémoire intraséquentielle (qui favorisera l'anaphore pronominale), d'autre part, le rôle important du locuteur (la réorientation de la visée référentielle favorisera l'anaphore nominale).

\subsection{Mémoire intra-séquentielle : succession de répliques sans dispersion du groupe de locuteur}

\subsubsection{En DD}

Il existe une mémoire référentielle qui se déroule tout au long des séquences dialogales et qui évite à chaque changement de locuteur de réintroduire le référent-thème sous une forme nominale. Dans ce cas, une forme pronominale est suffisante, ce malgré l'interposition d'autres chaînes anaphoriques ou référents.

15 Ainsi dans (1) où s'intercalent d'autres chaînes anaphoriques, sans ambiguïté référentielle, dans la chaîne anaphorique du référent-thème 'mari de la dame fraîchement accouchée', mentionné par le SN vostre mary. Chacun de ces deux référents intercalés apparaît sous une forme nominale proche mon mary ${ }^{18}$ mais le déterminant possessif de P1 les place en inter-définition avec leur énonciateur respectif. Le calcul inférentiel est donc réalisé en fonction du locuteur qui prend la parole. Chacune des chaînes se poursuit dans la réplique de son énonciateur par anaphore pronominale jusqu'au changement de locuteur.

(1) Et s'il avient qu'il faille aucune chose qui leur plaise, l'une des commeres dira a la dame: "Vroiement, ma commere, je me merveille bien, (...), dont vostre mary fait si petit compte de vous et de vostre enfant. Or regardez qu'il feroit si vous en aviez cincq ou six ! Il appert bien qu'il ne vous ayme gueres; si lui feistes vous plus grand honneur de le prendre qu'il avenist oncques a pié de son lignage. - Par mon serement, fait l'autre des commeres, si mon mary le me fasoit ainxin, je ameroye mieulx qu'il n'eust ne cul ne teste. - Ma commere, fait l'autre, ne lui acoustumez pas ainxin a vous lesser mectre soubz les piez, car $\mathbf{i l}$ vous en feroit autant ou pis l'annee a venir a voz aultres acouchemens. - Ma cousine, fait l'autre, je me merveille bien, veu que vous estes sage femme et de bon lignage et qu'il n'est pas vostre paroil, chacun le sceit, comment vous le lui souffrez; et $\underline{\mathbf{i l}}$ nous porte a toutes grant prejudice." Lors la dame respond et dit: "Vroiement, mes chieres commeres et cousines, je ne scey que faire et ne m'en scey chevir, tant est mal 
homme et divers. - $\underline{\mathbf{I}}$ est mal home ? dit l'une d'elles. Veez cy mes commeres qui scevent bien que, quant je fu mariee a mon mary, l'en disoit qu'il estoit si divers qu'il me tueroit. (...) Dieu mercy, j'ay tant fait que je puis dire ou faire ce que je veil, car la darraine parolle me demoura, soit tort ou droit. (...) Par madame sainte Caterine, ma commere, il seroit bien emploié qu'il vous crevast les yeulx. - Gardez, ma cousine, fait l'autre, que vous luy sonnez bien quant $\underline{\mathbf{i}}$ sera venu." Ainxin est il bien gouverné, le pouvre home ! (QJM, Tierce joye, p. 19-20, 1. 44 à 90)

Ces interpositions référentielles dans la chaîne anaphorique du référent-thème principal 'vostre mary' n'entraînent cependant pas, à la reprise de cette dernière, d'anaphore nominale. Cela est inattendu d'après les règles de la combinatoire. Or, en DD, la dimension supplémentaire du changement de locuteur semble à même d'éviter l'anaphore nominale du référent de la chaîne anaphorique principale comme si la reprise des données référentielles initiales se faisait "automatiquement" dans la mémoire immédiate du lecteur (schéma 1).

\begin{tabular}{|c|c|c|c|}
\hline \multirow{4}{*}{$\begin{array}{l}\text { Le référent et l'antécédent qui le représente } \\
\text { sémantico-référentiellement. }\end{array}$} & \multirow{4}{*}{$\begin{array}{l}= \\
\text { Thème }\end{array}$} & $\begin{array}{l}\text { Rhème } 1 \\
\text { Opinion } 1\end{array}$ & $\begin{array}{l}\text { Interlocuteur } \\
1\end{array}$ \\
\hline & & $\begin{array}{l}\text { Rhème } 2 \\
\text { Opinion } 2\end{array}$ & $\begin{array}{l}\text { Interlocuteur } \\
2\end{array}$ \\
\hline & & $\begin{array}{l}\text { Rhème } 3 \\
\text { Opinion } 3\end{array}$ & $\begin{array}{l}\text { Interlocuteur } \\
3\end{array}$ \\
\hline & & $\begin{array}{l}\text { Rhème } 4 \\
\text { Opinion } 4\end{array}$ & $\begin{array}{l}\text { Interlocuteur } \\
4\end{array}$ \\
\hline
\end{tabular}

SCHÉMA 1 : Mémoire intra-séquentielle en DD

17 Ainsi, en DD, la continuité référentielle semble permettre une confrontation d'opinions concernant le même référent-thème ce que dénote l'absence d'anaphore nominale à chaque changement de locuteur. Nous pouvons donc conclure à une mémoire référentielle du groupe de locuteurs pour une même séquence dialogale puisque la coréférentialité est assurée. Nous observons les mêmes conclusions pour les 5 passages de DD relevés dans les Chroniques ${ }^{19}$.

\subsubsection{En DI}

En DI, les expressions anaphoriques des locuteurs subissent un traitement spécifique : ils apparaissent généralement sous forme pronominale (P3 ou P6). Nous n'observons donc que les référents-thèmes.

\begin{tabular}{|l|l|l|l|l|}
\hline \multicolumn{2}{|l|}{} & QJM & Chroniques $^{\text {Mémoires }}{ }^{20}$ \\
\hline \multirow{2}{*}{$\begin{array}{l}\text { Extraits avec 1ère mention du référent suivi } \\
\text { d'une chaîne anaphorique avec } \\
\text { reprise dans la seconde réplique } \\
9 \text { séquences }^{21}\end{array}$} & Forme nominale & & $1^{22}$ & $1^{23}$ \\
\cline { 2 - 5 } & $\begin{array}{l}\text { Forme } \\
\text { pronominale }\end{array}$ & & 4 & 3 \\
\cline { 2 - 6 }
\end{tabular}

9 séquences $^{21}$ 
Les séquences étudiées montrent qu'il existe bien une mémoire intra-séquentielle qui permet poursuivre leur chaîne anaphorique par anaphore pronominale, d'une réplique à la suivante, malgré les changements de locuteurs, comme dans (2).

(2) Et envoia li rois de France des prelas de France et des chevaliers pour tretiier as Flamens que il vosissent venir dalés leur signeur le conte et faire a lui ce que il devoient, car voirement estoit li jones contes de Flandres en celle assamblee dou roi. Li Flamenc li remanderent par ses gens meismes que il n'avoient point de signeur, puisque $\underline{\mathbf{i}}$ se absentoit de euls et ne les voloit croire, ne que pour li il ne feroient riens, ne des rentes et revenues de Flandres, il n'en porteroit nulles; et se avoir les voloit, il les venist bellement et courtoisement despendre ou pais, et ouvrer par lor consel, mais il n'avoit pas encores bien conmenchiet; et se il voloit perseverer en ces opinions, il trouveroit les Flamens plus durs et plus hausters que onques n'euist fait son pere. (Chro., livre I, chap. 248, p. 822-823, 1. 89-105 : le DI est surligné en gris).

Les cas d'anaphore nominale ${ }^{24}$ au changement de locuteur dans la seconde réplique, peuvent être expliqués par l'application de la règle de concurrence référentielle ou un phénomène d'écho stylistique.

\subsection{La dimension du locuteur (observable en DD uniquement pour notre corpus ${ }^{25}$ )}

\subsubsection{Les facettes lexicales d'un même référent-thème selon l'opinion de chaque locuteur}

Malgré une mémoire intra-séquentielle, les différentes opinions émises respectivement par les locuteurs sur le référent-thème peuvent facultativement être accompagnées d'une redéfinition ${ }^{26} \mathrm{du}$ référent-thème (qui passe par une renomination sous une forme lexicale différente) véhiculant une réorientation de la visée référentielle concernant le référent en rapport avec les informations en relation avec la nouvelle opinion.

Dans (3), le référent-thème 'mari de la dame' est redéfini à cinq reprises, au gré des changements de locuteur.

(3) [rencontre entre la chambrière de la dame et le galant convoité pour ses largesses]

Il vient a elle et la salue et elle lui. «Quelles nouvelles, dit il, Jouhanne m'amie?

Que fait vostre maistresse?

- Par ma foy, fait elle, el est a l'oustel bien pensive et bien courrocee.

- Et de quoy, fait il, m'amie?

- Par ma foy, fait elle, monseigneur est si mal home que elle a trop mal temps.

- Ha a, fait il, mauldit soit il, le villain chutrin!

- Amen! fait elle, car nous ne pouons durer avecques lui en nostre meson.

- Or me dites, Jouhanne, que elle vous a dit.

- Par ma foy, fait elle, je lui en ay parlé, mes el ne s'i accorderoit jamés, car elle a si grant paour de son seigneur que c'est merveilles et a affaire a ung si mal home! Et si elle le vouloit ore, si ne pourroit elle, tant est gardee de son pere et de sa mere et de touz ses freres. Je cuide que la pouvre femme ne parla oncques puis a $\underline{\text { home que je demoure avecques elle, [...] elle ne vous reffuseroit pas pour nul aultre }}$ .(...) 
- Par mon serement, fait elle, le meilleur sera que vous parlez a elle, et il est bien a point, car son mary l'a reffusee d'une robe (...)» (QJM, Quinte joye, p. 43, 1. 338 à 369)

dialogales qui présentent des modifications de visée référentielle concernant le référentthème évoqué et pour chacune d'elles le changement de visée référentielle induit une forme nominale pour la mention du référent ${ }^{30}$. Il en va ainsi dans l'exemple suivant où le référent 'une pension que le Roy vous donne' est repris dans la seconde réplique de DD sous les formes nominales l'argent que le Roy nous donne puis tribut en tournure attributive qui fusionne les deux aspects de la visée référentielle du même référent. Ces redéfinitions sont par ailleurs l'un des enjeux de cette séquence dialogale :

(4) Mons ${ }^{\mathrm{r}}$ de Nerbonne, [...] ouyt ceste parolle et luy [= l'ung de ces Angloys] dist : « Estiéz vous si simples de penser que le duc de Bourgongne n'eust grand nombre de telz gens? Il les avoit seullement envoyéz refreschir; mais vous aviéz si bon voulloir de retourner que six cens pippes de vin et une pension que le Roy vous donne vous ont renvoyé bien tost en Angleterre. » L'Anglois se courrouça et dist : "C'est bien ce que chascun nous disoit, que vous mocqueriéz de nous. Appellé vous l'argent que le Roy nous donne pension? C'est tribut ; et, par sainct George, vous en pourriéz bien tant dire que nous retornerions.» Je rompis la parolle et le convertis en mocquerie; mais l'Angloys ne demeura point content, et en dist ung mot au Roy, qui merveilleusement se courrouça audict seigneur de Nerbonne. (Mém ., livre 4, chap. 11, p. 323, 1. 6-15)

\subsubsection{Lorsqu'un locuteur devient thème de la conversation}

Sur cinq séquences de ce type, deux extraits, un dans les QJM et un dans les Chro., sont pertinents pour l'observation ${ }^{31}$. En (5), le passage d'un locuteur au statut de référentthème ne nécessite pas de forme nominale lorsque les locuteurs ont tous le même passé référentiel. Le référent mentionné en tant que locuteur par l'interpellation beau filzextrait du groupe référentiel référent-thème 'voz enfans et les miens' et redéfini par le SN mon filz aisné - reste passif en DD et revient au statut de référent-thème avec les anaphores pronominales, $i l$ et $l e$, dans la réplique suivante de la mère qui utilise ces $\mathrm{SN}$ comme base référentielle appartenant à la mémoire intra-séquentielle sans recourir à une autre forme nominale.

(5) Et quant la dame et ses enfans sont davant lui, come dit est, il dit a la femme : «M'amie, fait il, vous estes la chose du monde que je doy plus amer [...] et voz enfans et les miens se portent mal envers moy. -Et que voulez-vous que je face? fait la dame. [...] - Ha a, belle dame, lessés en ester les parolles, car je n'en ay plus que fere!» 
Le bon homme parle a son filz aisné : «Enten a moy, beau filz ! [...] Tu es mon filz aisné et seras mon principal heritier, si tu te gouvernes bien. [...] Car si tu faiz le contraire, je te jure par ma foy que je te feroy desplaisir et que tu ne joïras de chose que Dieu me ait donnee, et t'en prens garde ! - Et que voulez vous, fait la dame, qu'il vous face? L'en ne savroit comment vous servir. [...] taisiez vous en et ne le soustenez pas! Car c'est tourjours vostre maniere. » (QJM, Neufviesme joye, p. 74-75, 1. 78 à 120)

En revanche, dans (6) où le référent 'les six bourgeois de Calais' intervient en DD comme locuteur, la forme nominale ces honmes qui sont en vostre merchi accompagne leur retour au statut de référent-thème.

(6) «Tres chiers sires, vechi la representation de la ville de Calais a vostre ordenance. » Li rois se taisi tous quois [...] Chils siis bourgois se missent tantos en genouls devant le roi et dissent [...] : « Gentils sires et nobles rois, veés nous chi siis , qui avons esté d'ancesserie bourgois de Calais et grans marceans par mer et par terre, et vous aportons les clefs de la ville et dou chastiel de Calais et les vous rendons a vostre plaisir, et nous mettons en tel point que vous nous veés en vostre pure volenté, [...] grietés. Si voelliés de nous avoir pité et merchi [...].» [...] Adont parla li gentils chevaliers mesires Gautiers de Manni et dist: "Ha! gentils sires, voelliés rafrener vostre corage. [...] Se vous n'avés pité de ces honmes qui sont en vostre merchi, toutes aultres gens diront que ce sera grans cruaultés, se vous faites morir ces honestes bourgois qui de lor propre volenté se sont mis en vostre ordenance pour les aultres sauver. » (Chro., livre I, chap. 240, p. 846-848, 1. 151-187)

Cette forme nominale utilise, à la fois, la détermination d'une relative, qui sont en vostre merchi, qui fait écho aux expressions référentielles précédemment utilisées dans le cotexte $^{32}$ et le déterminant démonstratif, ces, anaphorique et pointant déictiquement les entités référentielles en situation extra-linguistique.

Ces deux extraits ne permettent pas de tirer des conclusion générales. Cependant, selon nos observations, il semble que lorsqu'un référent passe du statut de locuteur à celui de référent-thème, il puisse être repris directement par anaphore pronominale sans anaphore nominale intermédiaire s'il est resté passif en DD. En revanche, lorsque ce locuteur est intervenu en DD, l'anaphore nominale est privilégiée suite à son changement de statut.

\subsection{Bilan intra-séquentiel}

En DD, nous avons pu constater qu'il une mémoire intra-séquentielle qui évite de reprendre un référent-thème sous forme nominale d'un locuteur à l'autre. Toutes les séquences dialogales présentant au moins deux répliques et une chaine anaphorique qui s'y étend, sont susceptibles de vérifier cela. Par ailleurs, le fait même qu'une seule séquence dialogale $\mathrm{du}$ corpus puisse procéder ainsi sans ambigüité référentielle, souligne l'existence de cette mémoire intra-séquentielle car si tel n'était pas le cas, les reprises pronominales ne seraient pas possibles. Ce bilan est appuyé également par le fait que les locuteurs peuvent au cours des répliques successives émettre des opinions sur le même référent-thème tout en le mentionnant par anaphore pronominale.

31 Néanmoins, ces opinions peuvent parfois être associées à l'utilisation d'une forme nominale différente de celle ayant servi à introduire le référent-thème. On parle alors de redéfinition du référent-thème qui donne à appréhender ce référent sous une visée référentielle différente. 
32 En DI, la mémoire intra-séquentielle se manifeste aussi par la possibilité de reprendre le référent-thème par anaphore pronominale en première mention de la seconde réplique de la séquence dialogale sans qu'il soit nécessaire de revenir à une mention nominale du référent-thème pour l'identifier. En revanche, en DI, l'influence de la visée du locuteur sur l'expression anaphorique du référent-thème (pas de redéfinition du référent-thème) n'a pu être observée.

\section{Relations inter-séquentielles ${ }^{33}$}

\subsection{En DD}

Le fonctionnement référentiel du DD nécessite que l'expérience référentielle vécue ou sensée être vécue par les locuteurs donne lieu à une mémoire référentielle valide entre ces locuteurs en présence. L'étude des expressions anaphoriques indique non seulement une mémoire référentielle intra-séquentielle, mais montre aussi que ce passé référentiel, en fonction de la stabilité du groupe de locuteurs, peut se voir réactivé au cours d'autres séquences dialogales, sans qu'il soit nécessaire de réintroduire le référent-thème par anaphore nominale à la séquence dialogale suivante. Nous ne présentons ici que les configurations que nous avons pu observer : le groupe de locuteurs reste identique ou un locuteur vient s'adjoindre au groupe au cours des séquences dialogales successives.

\subsubsection{Nombre et identité des locuteurs identiques d'une séquence dialogale à l'autre}

Trois occurrences dans les QJM $^{34}$, deux dans les Chroniques ${ }^{35}$ et aucune dans les Mémoires ${ }^{36}$ correspondent à cette configuration.

Selon notre hypothèse, le dialogue établit un passé référentiel mémorisé remis en service de manière implicite ${ }^{37}$ lorsque tous les locuteurs de la séquence dialogale initiale se retrouvent. Dans ce cas, les référents-thèmes de la séquence dialogale initiale restent valables et sont réactualisés dans - voire, enrichis par - les séquences dialogales qui suivent.

Pour exemple, dans les QJM, le passé référentiel établi entre la mère et sa fille au cours d'une première séquence dialogale ${ }^{38}$, fait état du référent-thème, 'l'écuyer à séduire'. Ce référent se voit repris par anaphore pronominale, il, à trois reprises dans la seconde séquence dialogale de (7), sans que l'anaphore nominale soit nécessaire.

(7) [bilan entre la dame et la jeune fille - enceinte avant le mariage - après une première approche de l'homme à séduire]

Quant vient aprés digner, la dame s'en va en sa chembre et demande a la fille: « Avant! fait elle. Dy moy comment tu as besongné. - Par mon serement, madame, fait elle, il ne me fine a journee de prier », et lui compte tout. "Or avant! fait elle, respons lui bien sagement et lui dy que l'en parle de te marier, mais que tu ne le vieulx point estre encore, et s'il se ouffroit a te prendre, mercie l'en et lui dy que tu m'en parleras et qu'il est l'omme du monde que tu ameroies mieulx. " (QJM, Onziesme joye, p. 87, 1. 189 à 197)

37 A l'inverse, dans les Chro., les deux passages montrent que le référent-thème est repris d'une séquence à l'autre sous forme nominale : dans l'une, il y a concurrence référentielle entre plusieurs référents-thèmes de mêmes caractéristiques ce qui explique la forme 
nominale, mais pas dans l'autre (8) où le référent-thème 'ceux de Calais' est mentionné en seconde séquence dialogale par l'anaphore nominale chil de Calais.

(8) [Il s'agit d'une entrevue entre le Roi d'Angleterre, Messire Gautier de Manni et les barons réunis]

Qant li rois d'Engleterre entendi ces nouvelles, il fist venir mesire Gautier de Manni devant lui. Qant il fu venus, il li dist: " Gautier, alés veoir que ces gens de Calais voellent dire: il me font requerre par lor chapitainne que je envoie parler a euls. " Mesires Gautiers respondi et dist: " Sire, volentiers. " (Chro., livre I, chap. 253, p. $835,1.22-25)$

li rois li demanda: " Mesire Gautier, que dient chil de Calais? " - " Tres chiers sires, respondi li chevaliers, il se voellent rendre, (Chro., chap. 254, p. 839, 1. 5-7)

Malgré (8), les résultats obtenus dans les QJM, (7) et autres occurrences, confirment qu'il existe une mémoire inter-séquentielle. Dans le cas contraire, aucune anaphore pronominale ne serait jamais possible d'une séquence dialogale à l'autre sans provoquer une rupture de continuité référentielle. Ainsi, si le groupe de locuteurs reste identique d'une séquence dialogale à l'autre, le référent-thème peut être - et non, "doit" être - repris par anaphore pronominale à l'entrée de chaque séquence dialogale suivant la séquence initiale ou précédente, sans que l'anaphore nominale ne soit nécessaire.

Ainsi, nous pourrions schématiser la mémoire inter-séquentielle de la façon suivante (schéma 2).

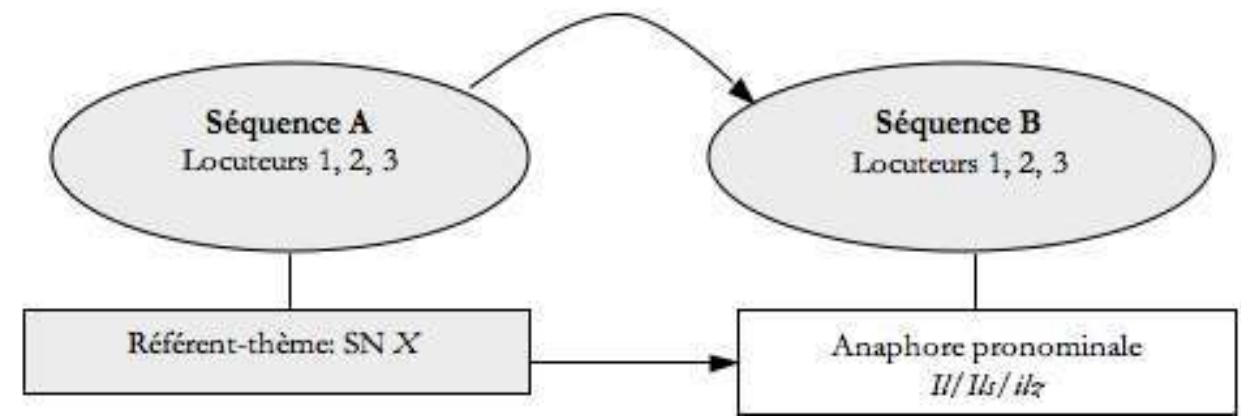

SCHÉMA 2 : Configuration 1 : Stabilité du groupe de locuteur initial et mémoire inter-séquentielle

\subsubsection{Lorsque s'adjoignent d'autres locuteurs au groupe de locuteurs préalablement concerné}

Lorsqu'un locuteur se greffe à un groupe de locuteurs ayant déjà un passé référentiel établi au cours de séquences dialogales antérieures, ce nouveau locuteur ne bénéficie pas de leur passé référentiel de sorte que pour suivre la conversation il doit en être informé : soit par lui-même en essayant de rassembler les éléments référentiels qui lui font défaut, soit à l'aide des autres locuteurs qui ré-instancient ce passé référentiel ainsi que les référents-thèmes qu'il contient qui apparaissent alors sous forme nominale.

Nous serions donc dans une configuration inter-séquentielle schématisée ainsi (schéma 3): 


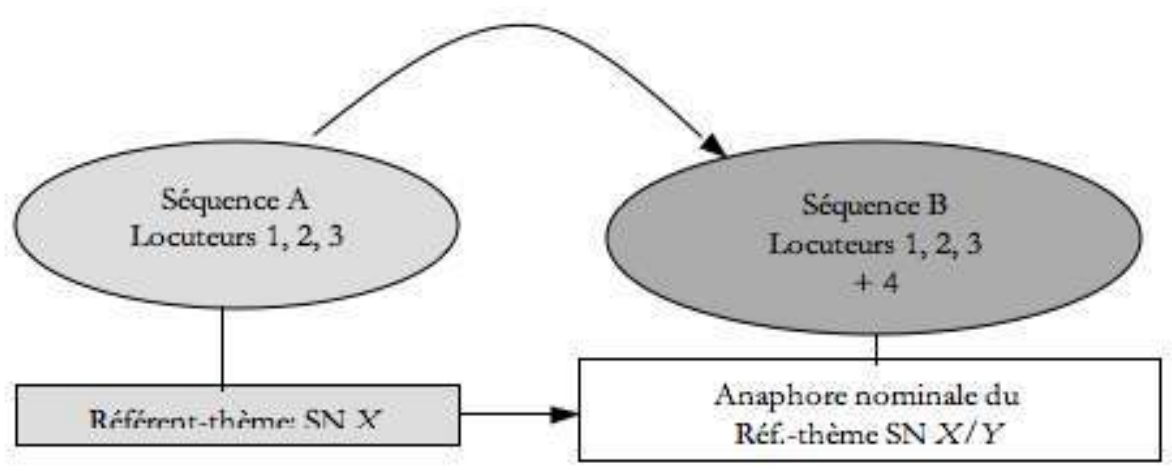

SCHÉMA 3 : Configuration 3 : Augmentation du groupe de locuteurs initial et mémoire inter-séquentielle

$$
\begin{aligned}
& \text { jouent un rôle important et rappellent les données référentielles nécessaires au nouveau } \\
& \text { locuteur (et parfois au lecteur). Malgré ce rappel mémoriel par le biais du récit ou du DI, } \\
& \text { le DD présente les référents-thèmes sous forme nominale suite à l'introduction du } \\
& \text { nouveau locuteur ce qui permet la remise à niveau du passé mémoriel pour le nouveau } \\
& \text { locuteur }{ }^{40} \text { comme dans l'exemple (9). }
\end{aligned}
$$

On relève une occurrence de ce type dans chaque texte ${ }^{39}$ et, pour chacune, le récit ou le DI

(9) Et retournerent chil doi chevalier sus lors logeis, ensi que tous desconfis, et furent sus un estat que de tantos departir de la et retourner arriere, qant evous descendu et venu entre euls un vaillant cevalier breton bretonnant, qui se nonmoit messires Garniers de Quadugal, [...]. Sitretos que il fu venus, li compagnon en orent grant joie, mais nonobstant sa venue, encores se voloient $\underline{\underline{i l s}}$ departir de la place, et ne se tenoient pas bien a aseguret. Qant messires Garniers de Quadugal les vei en cel esfroi, si leur demanda que il lor falloit. Li doi chevalier qui retourné estoient de la besongne, li recorderent sus briefs paroles com $\underline{\mathbf{i l}}$ avoient cevauchiet devant la Roce Dorient et conment il avoient esté ruet jus, et estoit demorés prisonniers mesires Thomas d'Agourne et encores pluisseurs autres chevaliers et esquiers: " Et ne veons point de rescouse en cela, et pour ce, nous volons nous retraire et retourner en Hainbon. Se nous perdons la Roce Deurient pour celle fois, une aultre fois venra que nous le recouverons. Il n'a pas ..II.. mois que nous le conquesimes: une fois desous et l'autre desus, ce sont li estat de gerre. " Qant messires Garniers les ot oi parler, il fu moult esmervilliés et [...] dist: " Biau signeur et mi compagnon, metés aultre arroi et ordenance en vous, et me creés de ce que je vous dirai, et grans biens vous en venra. Nous ferons armer et monter a cheval tous ceuls qui chevaus ont; et cheuls de piet nous les ferons sievir. Vous dites que il n'i a que deus petites lieues de chi en l'oost des François. Nous les courrons sus de grande volenté; il quident avoir tout achievé [...] il sont si raempli de glore, pour tant que il vous ont ruet jus, que il ne font point de gait, et sont, par le parti que je vous di moult legier a desconfire et a ruer jus. " (Chro., livre I, chap. 247, p. 814-815, 1. 142-181)

Dans (9), le nouveau locuteur messires Garniers de Quadugal vient s'adjoindre au groupe chil doi chevalier. Sa remise à niveau mémorielle passe par un premier DI (surlignement gris clair) où les référents-thèmes apparaissent sous forme nominale la Roce Dorient, mesires Thomas d'Agourne, pluisseurs autres chevaliers et esquiers; suivi d'un DD où le référent-thème 'Roce Dorient' apparaît également sous forme nominale ; puis par un discours rapporté en DD de la part du nouveau locuteur (surlignement gris foncé). Ce discours rapporté valide comme acquisition mémorielle du nouveau locuteur, les données référentielles d'un DD implicite et comble les lacunes mémorielles du lecteur ${ }^{41}$ avec l'utilisation de formes nominales pour les référents-thèmes. Les chaînes anaphoriques des référents-thèmes se poursuivent ensuite en DD par anaphore pronominale. 
Ainsi, la remise à niveau de la mémoire inter-séquentielle peut passer par le DI ou le DD voir par un discours rapporté en DD. Mais quel que soit le mode discursif les référents-thèmes seront repris par anaphore nominale.

\subsection{En DI}

Seules les Mém. présentent des associations inter-séquentielles pour le DI. Pour deux d'entre elles, le groupe de locuteurs reste identique d'une séquence à l'autre ${ }^{42}$, la troisième ${ }^{43}$ met en relation deux groupes de locuteurs dont un noyau reste identique d'une séquence dialogale à l'autre. Seul ce dernier extrait (10) permet d'observer l'existence (ou non) d'une mémoire inter-séquentielle. Ici, le messager de la première séquence dialogale est relayé par un autre messager dans la seconde séquence dialogale mais les différents messagers possèdent le même passé référentiel, celui de la première séquence dialogale.

(10) Comme il disnoit, on luy vint dire soubdainement que le marquis de Montagu, frere dudict conte, et quelque autre, estoient montéz a cheval et avoient faict crier a tous leurs gens : "Vive le Roy Henry! ” De prime face ne le creut pas ; mais incontinent y envoya plusieurs messaiges, et s'arma et mist des gens aux barrieres de son logis pour le desfendre. Il avoit la avecques luy ung saige chevalier appellé Mons $^{\mathrm{r}}$ de Hastingues, grand chambellan d'Angleterre, [...]. Ung aultre y avoit, appellé monsr d'Escalles, frere de la femme dudict roy Edouard, et plusieurs bons chevaliers et escuyers, qui tous congneurent que la besongne alloit mal; car les messagiers rapporterent que ce que avoit esté dict au roy estoit veritable, et s'assembloient pour luy venir courre sus. (Mém., livre III, chap. 6, p. 243, 1. 5-27 : le DI est surligné en gris clair, les référents-thèmes surlignés en gris foncé)

Cette mémoire référentielle inter-séquentielle est appuyée par l'anaphore "zéro" du verbe s'assembloient (P6) qui a pour actant 1 , le référent-thème mentionné dans la première séquence dialogale sous la forme nominale, le marquis de Montagu, frere dudict conte, et quelque autre. Notons également une anaphore contextuelle marquée par l'anaphore nominale, ce que avoit esté dict au roy, qui réfère au contenu sémanticoréférentiel de toute la première séquence dialogale de DI ainsi qu'au contenu sémanticoréférentiel de la séquence dialogale de DD qui y est enchâssée.

Ainsi, l'existence d'une mémoire référentielle inter-séquentielle en DI est vérifiée.

\subsection{Bilan inter-séquentiel}

Outre une mémoire intra-séquentielle, le DD présente une mémoire inter-séquentielle vérifiée par la possibilité de reprise par anaphore pronominale d'un référent-thème d'une séquence dialogale à l'autre si le groupe de locuteurs reste identique entre les séquences ${ }^{44}$ . L'ajout d'un nouveau locuteur induit à l'inverse une remise à niveau mémorielle passant par la mention sous forme nominale des référents-thèmes des séquences dialogales antérieures.

En DI, les référents-thèmes sont traités comme en récit selon les règles syntacticosémantiques de la combinatoire alors que les référents-locuteurs sont traités comme en $\mathrm{DD}$, toujours présentés sous forme pronominale et dégagés des règles de la combinatoire. Le DI, présente également les mêmes spécificités que le DD pour ce qui est de l'existence d'une mémoire intra-séquentielle comme d'une mémoire inter-séquentielle permettant de ne pas reprendre les référents-thèmes sous forme nominale à l'entrée d'une séquence 
dialogale. A ces différents titres, le DI présente donc un fonctionnement double : adoptant à la fois les fonctionnements du récit et préservant les spécificités du DD. Ce qui lui confère le rôle de passerelle référentielle entre le récit et le DD.

\section{Passerelles référentielles entre récit et $\mathrm{DD}$ : le rôle du DI(/DIL $\left.{ }^{45}\right)$}

Ce qui fait la particularité du DI, c'est sa relation syntactico-valentielle avec le récit et sa relation mémorielle avec le DD.

\subsection{Relations référentielles entre récit et DI}

En effet, le DI en proposition subordonnée conjonctive régie par un verbe de pensée ou de parole, voit sa distribution valentiello-référentielle entrer en relation avec celle du verbe qui l'enchâsse ${ }^{46}$ (voir tableau 2). Ainsi, le référent-thème en première mention dans le DI sauf rares exceptions explicables par la mémoire intra ou inter-séquentielle - est repris :

- sous forme nominale, s'il n'est pas mentionné dans la régissante du DI ou ses coordonnées

- sous forme pronominale, s'il est mentionné dans la régissante du DI ou ses coordonnées comme dans (11) avec 'son adversaire, Carle de Blois' repris par le pronom régime le en DI.

(11) Mais la ditte contesse de Montfort fu trop grandement resjoie de la prise de son adversaire, Carle de Blois, car elle imagina que sa guerre en seroit plus belle, et que li rois d'Engleterre le vodroit avoir et le acateroit au chevalier englois qui pris l'avoit. (Chro., livre I, chap. 247, p. 815, 1. 153 : DI surligné en gris clair, référents-thèmes surlignés en gris foncé)

\begin{tabular}{|c|c|c|c|c|c|c|c|}
\hline & \multirow[t]{2}{*}{$1^{\circ}$ mention } & \multicolumn{2}{|c|}{$\begin{array}{l}\text { QJM } \\
13 \\
\text { extraits }\end{array}$} & \multicolumn{2}{|c|}{$\begin{array}{l}\text { Chroniques } \\
27 \\
\text { extraits }\end{array}$} & \multicolumn{2}{|c|}{$\begin{array}{l}\text { Mémoires } \\
42 \\
\text { extraits }\end{array}$} \\
\hline & & & total & & total & & total \\
\hline \multirow{2}{*}{$\begin{array}{l}\text { Le référent n'est pas mentionné en régissante } \\
\text { enchâssant le DI ou dans ses coordonnées }\end{array}$} & nominale & 8 & \multirow{2}{*}{9} & 23 & \multirow{2}{*}{24} & 34 & \multirow{2}{*}{36} \\
\hline & pronominale & $1^{47}$ & & $1^{48}$ & & $2^{38}$ & \\
\hline \multirow{2}{*}{$\begin{array}{l}\text { Le référent est mentionné en régissante } \\
\text { enchâssant le DI ou dans ses coordonnées }\end{array}$} & nominale & 0 & \multirow{2}{*}{5} & $1^{49}$ & \multirow{2}{*}{6} & 0 & \multirow{2}{*}{7} \\
\hline & pronominale & 5 & & 5 & & 7 & \\
\hline
\end{tabular}

TABLEAU $n^{\circ} 2$ : Première mention du référent-thème en DI

52 En revanche, le DI qui répond à la règle syntactico-valentielle, se trouvant dans une proposition subordonnée conjonctive ${ }^{50}$ (frontière de sortie étanche ${ }^{51}$ ) ne permet pas à sa distribution valentiello-référentielle d'influer sur le choix de l'expression anaphorique des référents placés sur les rôles valentiels du verbe de la proposition non régie qui suit en récit.

Il peut donc assimiler les données référentielles qui le précèdent en récit mais pas les faire interférer avec celles qui viennent ensuite en récit. Il entretient donc avec le récit 
une relation extra-référentielle ${ }^{52}$. Il en va ainsi pour (12), où en DI le référent 'Jehan de Hatecele' est actant 1 des verbes peuist, venist et menast sous la forme pronominale, il, ainsi que par anaphore "zéro" à travers la P3 du verbe menast.

(12) Et escripsi tantos li rois [= le roi d'Engleterre] a mesire Jehan de Hatecele et li manda que, dou plus tos que il peuist, $\underline{\mathbf{i}}$ le venist veoir devant Calais et menast messire Carle de Blois son prisonnier en Engleterre. Li cevaliers obei as lettres dou roi sen signour, (Chro., livre I, chap. CCXLVII, p. 818, 1. 271-275: le DI est surligné en gris foncé, la proposition conjonctive est surligné en gris clair)

Or à la sortie du DI, ce référent reste actant 1 du verbe obei du récit. Si la distribution des verbes du DI influait sur celle du récit qui suit, le référent 'mesire Jehan de Hatecele' actant 1 du verbe obei, aurait dû être repris par anaphore pronominale pour marquer ce maintien valentiello-référentiel. Or il apparaît repris par anaphore nominale, Li cevaliers, ce qui indique que :

- le changement d'identité référentielle de l'actant 1 entre le verbe manda - actant 1 , li rois du récit précédant le DI en proposition non régie et le verbe obei en proposition non régie du récit suivant le DI est pris en compte ;

- le maintien du référent 'mesire Jehan de Hatecele' come actant 1 des verbes successifs entre le DI et le récit n'est pas pris en compte.

Cela s'applique du DI vers le récit de sorte que la distribution valentiello-référentielle des verbes en DI ne peut influer sur celle du verbe de récit qui suit le DI. Cela concerne autant les référents-locuteurs que les référents-thèmes. Cette étanchéité valentiello-référentielle entre DI et récit est dû à la catégorie grammaticale de la proposition conjonctive qui contient le DI. En effet, les propositions subordonnées conjonctives ont une frontière de sortie étanche ${ }^{53}$ qui implique que la continuité valentiello-référentielle est établie entre la proposition principale la conjonctive de DI et la proposition principale qui enchâsse la conjonctive de DI et la proposition qui suit la ou les proposition(s) conjonctive(s) supportant le DI sans tenir compte des données référentielles et valentiello-référentielles du DI.

\subsection{Relations référentielles entre récit et $\mathrm{DD}$ : le rôle du $\mathrm{DI} / \mathrm{DIL}^{54}$}

Intuitivement, nous savons que la continuité référentielle est un tout et ne se borne pas aux limites du DD ou du DI. Cette étude montre l'importance considérable du DI pour la continuité référentielle. Le DI rapporte un DD soit mot pour mot, soit en synthétisant une situation de dialogue antérieure au DD qui suit, soit encore en suggérant des paroles ou des pensées servant de préambule à une situation de dialogue. Dans ces trois cas, il a un rôle référentiel essentiel : il permet la mise en place de bases référentielles nécessaires à la compréhension du DD qui va suivre et, intégré au récit, il assure alors une passerelle référentielle du récit au DD.

\subsubsection{Le DI/DIL précède le $\mathrm{DD}$ : Porosité référentielle entre récit et $\mathrm{DD}$}

57 De par sa fonction qui consiste à enchâsser un DD dans le récit et ses propriétés référentielles, le DI permet de passer du récit au DD en assurant une continuité référentielle entre ces deux milieux discursifs : c'est pourquoi nous l'avons baptisé "zone référentielle tampon". Dans les QJM, il s'acquitte de ce rôle de deux manières : soit comme creuset du passé référentiel nécessaire au DD qui suit soit comme passeur référentiel assurant une continuité référentielle du récit au DD. 


\subsubsection{DI : Creuset du passé référentiel nécessaire au DD qui suit.} eu lieu avant l'apparition du DD en mentionnant les référents-thèmes qui seront nécessaires à la continuité référentielle en DD. Cette spécificité se manifeste à la fois avec des DI annoncés par des verbes de "dire" ou des verbes de "demande" ou d'énonciation volitive - pas de verbe de "pensée". Le DI a pour rôle quelque soit le verbe qui ouvrira le DD de poser les bases nécessaires à la continuité référentielle du DD qui suit et réalise alors une transition douce entre le récit et le DD en associant leurs données référentielles. En fonction du type de verbe ouvrant le DD, le DI soit réalise une synthèse de faits, soit présuppose un questionnement en préambule du DD.

ter un verbe de "dire" 55 , le DI rapporte mot pour mot un DD supposé. Ainsi dans (13), il fait figure de "zone tampon" entre le récit et le DD et permet, à l'ouverture du DD, de ne pas utiliser l'anaphore nominale pour les référents-thèmes mais de débuter en DD par l'anaphore pronominale ilz du groupe référentiel 'ces seigneurs [...] amis',

(13) Il va devers la dame et lui dit que ces seigneurs, qui sont ses parens et ses especiaulx amis, l'onst moult demandee, si la prie moult doulcement que el les vienne veoir et les festier et faire bonne chiere. «Et que iroi ge faire? fait elle. M'amie, je vous prie que vous y venez pour l'amour de moy. - Certes, fait elle, je ne iroy point. $\underline{\mathbf{I l z}}$ sont tropt grans maistres et $\underline{\mathrm{ilz}}$ prisent rien pouvres femmes. » (QJM, Sixiesme joye, p. 52-53, 1. 127 à 135) ;

de même en (14) où le référent "roy d'Angleterre » est introduit en DI sous forme nominale puis y est repris par anaphore pronominale ou zéro et apparaît directement sous forme pronominale en DD : $\underline{\mathbf{I}}$ ne veult vivre sinon en ses dissimulations, et entretenir chascun, et faire son prouffict.

(14) Le Roy [...] feist appeler cest herault et luy dist qu'il sçavoit bien que le roy d'Angleterre ne venoit point a sa requeste, mais y estoit contrainct tant par le duc de Bourgogne que par les communes d'Angleterre, et qu'i pouvoit bien veoir que ja sa saison estoit presque passee; et que le duc de Bourgongne s'en revenoit de Nuz comme homme desconfit et pouvre en touteschoses; et que, au regard du connestable, il sçavoit bien qu'il avoit prins quelques intelligences avec le roy d'Angleterre, pour ce qu'il avoit expousé sa nyepce, mais qu'il le tromperoit. Et luy compta les biens qu'il avoit de luy, disant: « $\underline{\mathbf{I l}}$ ne veult vivre sinon en ses dissimulations, et entretenir chascun, et faire son prouffict. » Et dist audict herault plusieurs aultres belles raisons pour admonnester le roy d'Angleterre de prendre appoinstement avecques luy. (Mém., livre 4, chap. 5, p. 293, 1. 30-31)

Introduit par un verbe de "demande" ${ }^{56}$ ou d'énonciation volitive, le DI synthétise le questionnement et pose les bases référentielles préalables au DD qui suit. Ainsi en (14), le référent-thème du DD est présenté en DI sous la forme nominale le gallant et peut être repris, par anaphore pronominale, à cinq reprises en DD et une fois en DI (récit sous autorité d'un verbe d'énonciation volitive) : elle ameroit mielx qu'il fust pendu. L'anaphore pronominale marque donc la continuité référentielle entre récit, DI et DD.

(15) La pouvre femme compte a sa mere tout ce qu'il lui est avenu, mes el li dit que le gallant estoit entré d'aventure liens et que oncques mes n'y avoit esté, et que son mari l'avoit trouvé d'aventure parlant a elle sans aultre mal faire. Et sa mere li demande : «Que deable, fait elle avoit $\underline{\text { l }}$ affere avecques toy ? - Par Dieu, il est bien vroy qu'il m'avoit parlé deux ou troys foiz de cela, mes je l'en avoye bien reffusé, et

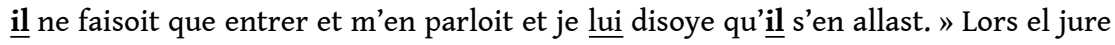
grans seremens que elle ameroit mielx qu'il fust pendu. Ou a l'aventure el lui confesse toute la maniere, quar la mere lui dit, [...] : « Certes, je me doubte qu'il y ait 
aultre chose, ne je ne t'en creroy ja qu'il eust ousé entrer en ta chambre s'il n'eust grant acointance a toy. [...]. (QJM, Quinziesme joye, p. 105-107, 1. 46 à 109 : DI surligné en gris) en DD.

(16) Quant ilz furent joinctz, celuy qui avoit esté sur l'arbre demanda a son compaignon par serment ce que l'ours luy avoit dict en conseil, qui si long temps lui avoit tenu le museau contre l'oreille. A quoy son compaignon luy respondit : « Il me disoit que jamais je ne marchandasse de la peau de l'ours jusques ad ce que la beste fust morte.» Et avecques ceste fable paia l'Empereur nostre homme, sans faire aultre responce, sinon en conseil (Mém., livre 4, chap. 3, p. 287, 1. 25-31)

\subsubsection{DI : Rôle de passeur référentiel du récit au $\mathrm{DD}^{57}$}

Ce rôle, plus complexe, du DI consiste à reprendre en DI par anaphore nominale ou pronominale, un référent déjà évoqué dans le récit, pour assurer un lien de coréférentialité avec le même référent-thème du DD qui suit. Il a alors un rôle de "passeur" ${ }^{58}$ référentiel du récit au DD.

(17) Lors soubvient au bon homme que, quant ung escuier du païs, qui est ung grant galant, vient liens, n'a rien espargné, et toutevoies le bon homme li a dit qu'

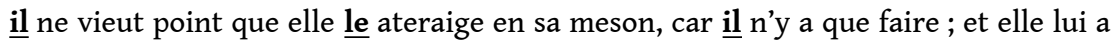

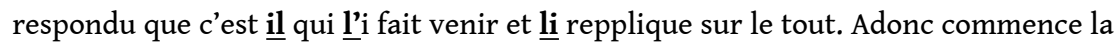
noise et a l'aventure la batra, mes il fera que foul. Si li dit le bon homme : «Par le salut que je actens a avoir, s'il avient que jamés le trouve ciens ne que vous parlez a $\underline{\text { lui, }}$ je vous feroy la plus courrocee que vous fustes oncques. -Par ma foy, fait elle, il ne m'en chauldroit s'il estoit pendu ! [...] suy. » (QJM, Sixiesme joye, p. 54-55, 1. 192 à 207)

Dans (17), le référent 'gallant' apparaît sous forme nominale en récit puis est repris par anaphore pronominale en DI, puis en DD : le DI assure donc la porosité référentielle entre le récit et le $\mathrm{DD}^{59}$.

\subsubsection{DIL : Rôle de passeur référentiel du récit au $\mathrm{DD}^{60}$}

Une des occurrences relevées présente un DIL qui précède le DD. Le référent-thème 'le roy d'Angleterre' y est introduit sous forme nominale, puis il est repris dans la séquence dialogale qui suit directement sous forme pronominale, avec le pronom démonstratif c' puis par les pronoms personnels il et luy.

(18) Comme le Roy se retira de ceste veue, parla a moy au long du chemin sur deux pointz. Il trouva le roy d'Angleterre si prest a venir a Paris que cela ne luy avoit point pleu, et disoit : " C'est ung tres beau roy ; il ayme fort les femmes : il pourroit trouver quelque affectee a Paris, qui luy sçauroit bien dire tant de belles parolles qu'elle luy feroit envye de revenir. »; et que ses predecesseurs avoient trop esté a Paris et en Normendie, et que la compaignee de l'amytié ne valloit rien deça la mer ; mais que dela la mer il le vouloit bien pour frere et pour bon amy. (Mém., livre 4, chap. 10, p. 317, 1. 7-17)

Le DIL présente combine les caractéristiques du récit et la faculté du DI à servir de creuset du passé référentiel nécessaire au DD. 


\subsubsection{Cas particuliers : quand le référent n'est explicite ni en DD ni en DI} occurrences ont été relevées : l'une d'entre elles révèle une mémoire intra-séquentielle, les deux autres révèlent une mémoire inter-séquentielle.

\subsubsection{Mémoire référentielle intra-séquentielle du DD au DI qui suit (une occurrence)}

(20) Et s'il pleut ou gelle ou grelle et le mary soit dehors, l'une d'elles dira ainxin: «Hellas! mon compere a maintenant mal endurer, qui est dehors! » Et l'autre respond qu'il n'y a force et qu'il est bien aise. (OJM, Tierce joye, p. 19, 1. 39-43)

Dans (20), le référent 'mary' est un référent-thème du DD et y apparaît sous la forme nominale mon compere. Il est ensuite repris par anaphore pronominale par $i l$, sujet $\mathrm{du}$ verbe, est, dans le DI qui prolonge le DD.

Dans les Mém., nous avons pu relever également deux occurrences ${ }^{64}$ dont celle citée en (18) où l'on constate que le référent 'roy d'Angleterre' continue à être repris sous la forme 
pronominale le dans le DI qui suit le DD (les deux faisant partie d'une même séquence dialogale).

(18) Comme le Roy se retira de ceste veue, parla a moy au long du chemin sur deux pointz. Il trouva le roy d'Angleterre si prest a venir a Paris que cela ne luy avoit point pleu, et disoit : « C'est ung tres beau roy ; il ayme fort les femmes : il pourroit trouver quelque affectee a Paris, qui luy sçauroit bien dire tant de belles parolles qu'elle luy feroit envye de revenir. »; et que ses predecesseurs avoient trop esté a Paris et en Normendie, et que la compaignee de l'amytié ne valloit rien deça la mer; mais que dela la mer il le vouloit bien pour frere et pour bon amy. (Mém., livre 4, chap. 10, p. 317, 1. 7-17)

Ainsi, ces extraits confirment qu'il existe une mémoire intra-séquentielle entre DD et DI et marquée par l'anaphore pronominale du référent-thème en DI.

\subsubsection{Mémoire référentielle inter-séquentielle du DD au DI}

Nous avons relevé deux occurrences ${ }^{65}$ en DI dans les QJM. Dans (21), le groupe de locuteurs du DD est 'la dame, le mari et le fils aîné'.

(21) Et quant la dame et ses enfans sont davant lui, come dit est, il dit a la femme : « M'amie, fait il [= le bon homme], vous estes la chose du monde que je doy plus amer [...] et voz enfans et les miens se portent mal envers moy. - Et que voulez-vous que je face? fait la dame. [...] - Ha a, belle dame, lessés en ester les parolles, car je n'en ay plus que fere!»

Le bon homme parle a son filz aisné : «Enten a moy, beau filz ! [...] Tu es $\underline{\text { mon filz }}$ aisné et seras mon principal héritier, si tu te gouvernes bien. [...] Car si tu faiz le contraire, je te jure par ma foy que je te feroy desplaisir et que tu ne joïras de chose que Dieu me ait donnee, et t'en prens garde ! - Et que voulez vous, fait la dame, qu'il vous face? L'en ne savroit comment vous servir. [...] Vous ne savez que vous demandez : n'estez vous pas bien aise ? - Or, belle dame, fait il, taisiez vous en et ne le soustenez pas ! Car c'est tourjours vostre maniere. "

Lors se departent et parlent la dame et le filz ensemble et dient qu'il est assoty. Et pour ce qu'il menace le filz, ilz dient qu'il est en voie d'empirer son heritage, qui n'y pourverra, et concluent ensemble que home du monde ne parlera plus avecques lui. Le filz vieult entrer en gouvernement plus que davant, quar la mere le soustient. Ilz s'en vont et dient a chacun que le proudomme est tourné en enfance, et travaille le filz a faire metre le bon homme en tutelle [...] (OJM, Neufviesme joye, p. 74-75, 1. 78 à 129)

L'ancien locuteur 'mari' devient le référent-thème du DI et y apparaît par anaphore pronominale, il, sujet du verbe est assoty et poursuit sa chaîne anaphorique sous forme pronominale (il, sujet de est et, lui, régime indirect de parlera). La première anaphore pronominale du DI, il est assoty, valide la continuité référentielle entre les deux séquences dialogales, DD et DI, dont les locuteurs ont même passé référentiel.

Dans la dernière séquence de DI (surlignée gris foncé) des locuteurs nouveaux, 'chacun', s'adjoignent au groupe 'mère-fils' d'où la forme nominale le proudomme pour la reprise du référent-thème 'bon homme' afin de combler les lacunes référentielles de ces nouveaux locuteurs.

\subsubsection{Bilan DD > DI : DI prolongation du DD}

77 Les tests montrent que, du DD au DI qui suit, lorsque les locuteurs ont le même passé référentiel d'une réplique ou séquence dialogale à l'autre, une mémoire référentielle existe; ainsi, l'anaphore pronominale permet de reprendre le référent-thème sans 
utiliser d'anaphore nominale préalable. La mémoire intra et inter-séquentielle sont donc vérifiées quel que soit le mode du dialogue, DD ou DI.

\section{Conclusion}

78 Aux vues de cette étude, le récit présente un fonctionnement que l'on retrouve en DD et en DI. Mais ces deux autres milieux ont également des spécificités de fonctionnement qui leur sont propres et qui se retrouvent de l'un à l'autre : en DI, un traitement particulier des référents-locuteurs et, en DD et DI, une mémoire intra et inter-séquentielle qui joue et s'appuie sur le choix des expressions anaphoriques à l'entrée des répliques ou séquences dialogales.

En outre, il apparaît que le DI/DIL présente un fonctionnement original sur le plan référentiel. Ce fonctionnement référentiel spécifique du DI/DIL fait de celui-ci un intermédiaire référentiel entre récit et DD puisqu'il peut prolonger la continuité référentielle du récit en son sein et la transmettre à un DD qui le suit, voire la poursuivre après le DD. Il joue donc un rôle de passeur référentiel qui lui propre pour la continuité référentielle.

\section{BIBLIOGRAPHIE}

M. Ariel (1990), Accessing Noun Phrases Antecedents, London, Routledge.

Cl. Blanche-Benveniste et alii (1984), Pronoms et syntaxe : l'approche pronominale et son application au français, S.ELA.F., Paris.

P. Bosch (1983), Agreement and Anaphora. A Study of the Role of Pronouns in Syntax and discourse, Academic Press, London.

Fr. Corblin (1985), Anaphore et interprétation des segments nominaux, thèse d'Etat, Université de Paris VII.

-, (1995), Les formes de reprise dans le discours, Anaphores et chaînes de référence, Presses Universitaires, Rennes.

F. Cornish (2000), « L'accessibilité cognitive des référents, le centrage d'attention et la structuration du discours: une vue d'ensemble ", Verbum, XXII, 1, p. 7-30.

E. Dupuy-Parant ${ }^{66}$ (2006), La continuité référentielle en moyen français : règles syntactico-sémantiques, thèse de Doctorat, Université du Maine, Le Mans.

- (2007), « Les expressions anaphoriques : fréquence et contraintes linguistiques en récit dans les chaînes anaphoriques du XIVème au XVème siècle » in Vanderheyden, A. \& alii (éds.) (2007), Texte et discours en moyen français, Actes du XIème Colloque international sur le moyen français, Turnhout, Brepols, p. 11-24.

- (2008), « Le verbe au cœur de la continuité référentielle : Unité structurante et maillage interverbal des systèmes valentico-référentiels », in B. Fagard \& alii (éds) (2008), Evolutions en français, 
études de linguistique diachronique, Actes du Colloque international Diachro-3, Paris, Peter Lang., vol. 86, p. 63-85.

E. Dupuy (2010), « La voix passive et les auxiliaires de voix en moyen français : Maillage interverbal des systèmes valentico-référentiels ", Description historique et contrastive des langues romanes , Actes du 25ème Colloque International de Linguistique et Philologie Romane, section 5, Innsbruck, Autriche, Editions Niemeyer.

- (2012), « Du récit au discours direct : “choix défini” des expressions anaphoriques en moyen français, Colloque international et linguistique », Anaphore et anaphorique : diversité des grammèmes, diversité des langues, Mont-Saint-Aignan : Presses Universitaires de Rouen et du Havre, coll. « Cahiers de l'ERIAC », p. 281-302.

E. Dupuy et L. Limousin (2015), « Pronoms relatifs lequel vs -qu- : conséquences pour la continuité référentielle des propositions relatives; Etude diachronique de l'ancien au moyen français », in Relatives et autres subordonnées - corpus, acquisition et didactique, Presses Universitaires de Rennes (PUR).

K. Ehlich (1982), Anaphora and deixis: Same, similar, or different. Speech, place and action. Studies in deixis and related topics, 315-338.

- (1983), Text und sprachliches Handeln. Die Entstehung von Texten aus dem Bedürfnis nach Überlieferung. na.

T. Givon (1989), Mind, Code and Context, Essays in Pragmatics, Lawrence Erlbaum Associates, London.

B. Grosz et alii (1995), « Centering: a framework for modeling the local coherence of discourse », Computational Linguistics, 21, 2, p. 203-225.

J.-K. Gundel et alii (1993), « Cognitive status and the form of referring expressions », Language, 69, 2, p. 274-307.

G. Kleiber (1991), Anaphore - deixis : où en sommes-nous ? L'information grammaticale, 51, 3-18.

G. Kleiber et alii (eds.) (1997), La continuité référentielle, Recherches linguistiques XX, Paris, Klincksieck.

- (2001), L'anaphore associative, PUF, Paris.

F. Landragin \& C. Schenedecker (dir.) (2014), Les chaînes de référence, Langages, 195.

L. Melis (1990), La voix pronominale : la systématique des tours pronominaux an français moderne, Paris, Duculot.

- (2003), La préposition en français, Paris, Ophrys.

E.-F. Prince (1981), Toward a Taxonomy of Given-New Information, in P. Cole (ed.), Radical Pragmatics, New-York: Academic Press, 223-255.

M. Riegel, J-Ch. Pellat, R. Rioul (1994), Grammaire méthodique du français, Paris, PUF, p. 614.

C. Schnedecker (1997), Nom propre et chaînes de référence, Recherches linguistiques, XXI, Paris, Klincksieck.

M. Walker (2000), « Vers un modèle de l'interaction du centrage avec la structure globale du discours ", Verbum, XXII, 1, p. 31-58.

A. Zribi-Hertz (1996), L'anaphore et les pronoms : une introduction à la syntaxe générative, Villeneuved'Ascq (Nord), Presses universitaires du Septentrion. 
- (1986), Relations anaphoriques en français : esquisse d'une grammaire générative raisonnée de la réflexivité et de l'ellipse structurale, Doctorat d'Etat, Paris 8, Lille, ANRT.

\section{NOTES}

1. La coréférence est un phénomène qui permet d'identifier à l'aide d'une expression référentielle suffisamment complète sémantiquement un référent mis en mémoire (directement ou indirectement) sans qu'il soit nécessaire de rechercher un contenu sémantique par le truchement une expression référentielle antérieure. Il s'agit d'un « rapport symétrique » comme l'explique F. Corblin (1995 : 31 infra). L'anaphore, en revanche, est un phénomène basé sur le fait que l'expression référentielle anaphorique révèle "une incomplétude et la nécessité de saturation référentielle » (Jokinen, $1992: 47-48$ ). Ainsi le " rapport [est] dissymétrique entre un terme, dit en français anaphorique, et un antécédent ou "source"[...] L'anaphore, relation de dépendance orientée, s'oppose à la coréférence, relation symétrique d'identité référentielle entre des termes interprétables indépendamment l'un de l'autre » (Corblin, 1995: 31). L'anaphore et la coréférence, lorsqu'il s'agit d'un même référent, sont complémentaires et elles permettent, par leur association, puisqu'elles concourent au déroulement d'une chaîne anaphorique - qui comporte, au sens où nous l'entendons, des expressions anaphoriques et coréférentielles d'assurer la continuité référentielle, c'est-à-dire la perception/l'identification du référent au fil du discours ou du texte.

2. Forme zéro ou "anaphore zéro" se dit de l'absence de mention du référent qui peut néanmoins être appréhendé à travers d'autres indices tels que la personne verbale lorsqu'il est en fonction de sujet ou d'objet (participes passés) ou la morphologie déterminative ou adjectivale qui peuvent donner des indications morpho-sémantiques sur le référent à sélectionner en contexte (exemple : j'ai choisi la rouge). Il faut notamment préciser que la morphologie verbale en MF n'est que peu ambigüe.

3. Quinze Joies de Mariage (fin XIV ${ }^{\text {ème }}$ siècle) : 5 premières joies (hors prologue) - éd. Rychner (1967) p. 6-48 et en totalité pour le DD (63 séquences dialogales des DD) ; les Chroniques de Froissart (vers 1400) : livre I, chap. CCXLVII à CCLVI inclus - éd. T. Diller (1972), p. 810-856 (20 séquences dialogales de DD autour du siège de Calais); Mémoires de Commynes (fin XV ème siècle) : livre III, chap. 1-8 -l'éd. Blanchard (2001) p. 217-257, additionné des livres 4-5-6 pour le DD (16 séquences dialogales de DD hors DR). Ont été ciblées les chaînes anaphoriques faisant appel au pronom personnel $\mathrm{Il} / \mathrm{ils} / \mathrm{ilz}$ - parfois à l'origine d'hésitations morphologiques et engendrant des stratégies référentielles visant à éviter un faux-sens - dans leur déroulement tout en y analysant toutes les expressions anaphoriques s'y relayant.

4. La comparaison dialectale est moins évidente car le texte des QJM a vu ses traits dialectaux du Nord Poitou lissés par l'éditeur (Rychner, 1963: XXXVII) et le texte des Mémoires est dans une langue du $\mathrm{XV}^{\mathrm{ème}}$ siècle qui ne présente déjà plus de traits dialectaux apparents (éd. Blanchard, 2001 : 62-63 et C Paris, BNF, ms., fr. 5063 - fin XV ème siècle). Pourtant, par opposition, les Chroniques sont de scripta picarde et certains relevés effectués sur l'usage de catégories de propositions syntaxiques révèlent une opposition fréquentielle entre les QJM et les Mémoires, d'une part, et les Chroniques, d'autre part (Dupuy-Parant, 2006 : chap. 2).

5. Dupuy-Parant, 2006, 2007, 2008 et Dupuy, 2012.

6. Pour nous, le terme de "redéfinition" associe le moyen utilisé, la "renomination", et la notion de réorientation de la visée référentielle sous-jacente à cette renomination. D'autres aspects de la représentation stéréotypique du référent sont alors visés en cas de redéfinition ou sont alors greffés au référent initial. 
7. L'anaphore lexicale infidèle, pour mémoire, est la reprise d'un référent déjà mentionné sous une forme nominale dont le nom tête est différent de celui initialement utilisé (cf. Riegel \& alii, 1994 : 614) : Le chien de mon voisin était très laid. Ce vieux cabot n'aimait pas non plus les gens.

8. Sont incluses les anaphores indirectes ou associatives (Kleiber, 2001) assez rares. Les redéfinitions, dans la majorité des cas, modifient la visée référentielle antérieure. Appliquées au référent en rôle d'actant 1 ou 2, elles assurent un apport informationnel nouveau pour le référent, très souvent lié au contexte situationnel.

9. Les réinitialisations évitent l'ambiguïté référentielle.

10. Les arguments d'un même verbe (non pronominal) ne peuvent avoir une même identité référentielle (Melis, 1990, 2003 ; Zribi-Hertz, 1996, 1986).

11. Le maintien d'un référent d'actant 1 en actant 1 est appuyé par l'anaphore pronominale ou zéro ; un changement d'identité référentielle dans la succession linéaire des actants 1 est marqué par l'anaphore nominale. Idem pour les actants 2 successifs (Dupuy-Parant, 2006, chap. 5).

12. Les propositions non régies, subordonnées temporelles et relatives en lequel reçoivent l'influence de la distribution valentiello-référentielle du verbe qui précède et influencent celle du verbe du suit. Les autres propositions subordonnées ne peuvent que recevoir l'influence de la distribution valentiello-référentielle du verbe qui précède (Dupuy et Limousin, 2015).

13. Ont été retenus tous les passages (42 séquences; voir références dans Dupuy-Parant, 2006 : 617 en note) de DI où la proposition conjonctive ou interrogative indirecte est régie par un verbe de parole ou de pensée (Rychner, 1970) qui génère des séquences qui ont les mêmes caractéristiques spécifiques que celles des séquences régies par un verbe de parole. Pour des questions de volume nous n'aborderons pas les séquences de DI enchâssées dans du DD ni de discours rapportés insérés en DI.

14. Ayant déjà fait l'objet d'une publication, l'étude du DI ne sera pas développée dans cet article, nous ne mentionnerons que les résultats importants de celle-ci (Dupuy, 2012).

15. Cf. note infra (15) et (16).

16. Une séquence dialogale correspondra à une succession de répliques en DD ou en DI sans interruption longue de récit (admises les didascalies, les incises ou les très courts passages de récit visant uniquement à introduire le DD qui suit). Les relations intra-séquentielles sont les relations référentielles qui auront été observées au sein d'une même séquence dialogale.

17. Seront considérées comme une succession de séquences dialogales formant un ensemble, les séquences dialogales au sein desquelles le thème de discussion est maintenu (de façon non exclusive) et pour lesquelles au moins une partie des locuteurs est commune (au moins deux locuteurs communs). Les relations inter-séquentielles sont les relations référentielles qui auront été observées entre une série de séquences dialogales successives partageant le même thème de discussion et un groupe de locuteur commun.

18. 'mon mary', (dans « si mon mary le me fasoit ainxin, je ameroye mieulx qu'il n'eust ne cul ne teste. ») et 'mon mary', (dans « quant je fu mariee mon mary, (...) droit. »

19. Références des cinq séquences dialogales comprenant plusieurs répliques dans le livre I des Chroniques de Froissart : Chapitre CCXLVII, p. 815, 1. 159-181; Chapitre CCLI, p. 830-831, 1. 44-74 ; Chapitre CCLIII, p. 836-838, 1. 31-94; Chapitre CCLIV, p. 839-841, 1. 5-71; Chapitre CCLV, p. 847-848, 1. 178-213. Les Mém. n'infirment pas l'influence du locuteur mais les extraits sont trop courts pour obtenir des résultats.

20. Uniquement à partir du livre 3 des Mém. (hors livre 4-5-6).

21. Quatre séquences ne sont pas répertoriées dans ce tableau car elles ne présentent pas de référent-thème dans leur première réplique : 1 dans les $Q J M, 2$ dans les Chro. et 1 dans les Mém.

22. Chro., livre I, chap. CCXLVII, p. 811, 1. 54-59 suivi de p. 811-812, 1. 59-65.

23. Mém., livre 3, chap. 3, p. 225, 1. 13-22 suivi de p. 225, 1. 23 - p. 226, 1.8.

24. Voir extrait cités dans les deux précédentes notes.

25. Corpus complet pour le DD. 
Pour le DI, corpus hors livres 4-5-6 des Mém. L'étude des livres 4-5-6 des Mém. pourraient révéler quelques passages de DI où l'opinion des locuteurs influerait sur le choix des formes nominales à la reprise $\mathrm{du} / \mathrm{des}$ référents-thèmes. Cette étude reste à faire.

26. En récit ou en discours, les redéfinitions ne sont pas nécessairement liées à un changement d'opinion (il peut aussi être question de référents évolutifs ou de perception du référent non évolutif sous un autre angle référentiel). Or en DD, nous n'avons pu noter que les changements d'opinion pour un même locuteur ou d'un locuteur à l'autre peuvent être accompagnés d'une redéfinition.

27. A l'inverse du récit qui, pour schématiser, ne fait état que d'un locuteur, le narrateur, et d'un interlocuteur passif, le lecteur.

28. Notons également que dans cet extrait, aucune ambiguïté référentielle n'est possible puisque le référent-thème 'monseigneur' est le seul référent-thème possédant ces caractéristiques référentielles. Il n'entre donc pas en concurrence référentielle avec les autres référents-thèmes co-présents : son pere et de sa mere et de touz ses freres et home.

29. Chro., livre I.: Chap. 247, p. 815, 1. 159-181; Chap. 251, p. 830-831, 1. 44-74; Chap. 253, p. 836-838, 1. 31-94 ; Chap. 254, p. 839-841, 1. 5-71; Chap. 255, p. 847-848, 1. 178-213.

30. Mém., livre 4, Chap. 11, p. 323, 1. 6-15; livre 6, Chap. 1, p. 424.

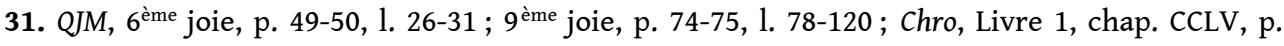
846-848, 1. 151-187 et Mém., livre 3, chap.8, p. 255, 1. 13-22.

32. euls siis honmes bourgois des plus notables de Calais, [...] viennent ichi [...] et de ceuls je ferai ma volenté; (Chro., livre I, chap. 254, p. 841, 1. 64-70)

33. Les séquences dialogales concernées sont celles séparées par un passage au récit qui implique une séparation du groupe de locuteurs jusqu'à leurs retrouvailles selon certaines conditions.

34. QJM : $11^{\text {ème }}$ joie, p. $87,1.189-197 ; 5^{\text {ème }}$ joie, p. $44,1.384-388\left(+5^{\text {ème }}\right.$ joie, p. $\left.41-42,1.293-332\right) ; 5^{\text {ème }}$ joie, p. 45, 1. 409-435.

35. Chro. : livre 1, chap. 253, p. 836-838, 1. 32-94 ; livre 1, chap. 255, p. 842, 1. 4-13 / livre 1, chap. 255, p. 845-846, 1. 115-128.

36. Les Mém. présentent le plus souvent de courtes séquences dialogales (une deux répliques) et le passé référentiel de ces séquences n'est pas réactivé pas réactivé dans des séquences dialogales ultérieures de DD ce qui ne permet pas de réaliser cette étude entre séquences de DD. Cependant, nous verrons que les séquences dialogales de DD des Mém. sont fréquemment liées à une séquence dialogale de DI qui précède, suit ou les deux le DD.

37. C'est-à-dire sans qu'il soit nécessaire de mentionner les référents-thèmes à nouveau sous forme nominale.

38. QJM, p. 84, 1. 64 à 101.

39. QJM, 15 ${ }^{\text {ème }}$ joie, p. 107, 1. 110-116 ; Chro., livre 1, chap. 247, p. 814-815, 1. 142-181; Mém., livre 3, chap. 8, p. 11-22.

40. Cela semble suggérer que le rappel mémoriel en récit ou DI est surtout destiné au lecteur - ce qui est logique puisque le texte est écrit pour le lecteur pas pour les personnages -, la reprise sous forme nominale dans le discours direct étant une manière de reproduire la réalité (fictive ou non) du DD.

41. Voir infra : Passerelles référentielles entre récit et DD : le rôle du DI/DIL.

42. Corpus étudié pour le DI dans les Mém. : Livre 3 : Mém., livre 3, chap. 6, p. 243, 1. 5-11 + Mém., livre 3, chap. 6, p. 243, 1. 20-27 ; Mém., livre 3, chap. 6, p. 238, 1. 17-23 + p. 246, 1. 15-20, 1. 5 + p. 249, 1. 10-14 + p. 255, 1. 23-31. Dans ces deux occurrences le test est impossible: pas de chaîne anaphorique ou de maintien du référent-thème d'une séquence dialogale à l'autre.

43. Mém., livre 3, chap. 6, p. 238, 1. 34-38 + p. 239, 1. 9-11.

44. Sur les conseils bienveillants de l'un de nos relecteurs, nous avons extrait du corps de l'article la configuration qui présenterait le départ ou l'absence d'un des locuteurs du groupe car nous n'avons relevé aucune occurrence présentant, en DD, cette configuration. Cependant, puisque 
nous avons pu établir qu'il existe une mémoire inter-séquentielle lorsque le groupe de locuteurs est identique d'une séquence dialogale à l'autre et que, selon toute vraisemblance, l'absence de l'un des locuteurs n'influe pas sur la mémoire collective des autres locuteurs présents, il semble que nous puissions émettre l'hypothèse (non vérifiée sur le corpus) que la réactivation ultérieure du passé mémoriel des autres locuteurs initialement présents ne posera pas de problème. Les référents-thèmes devraient pouvoir être réactivés par anaphore pronominale comme dans le cas de séquences dialogales successives présentant les mêmes locuteurs.

En revanche, si le locuteur absent de l'une des séquences dialogales revenait dans le groupe subséquemment, sa réintégration pourrait nécessiter une remise à niveau mémorielle, peut-être sous la forme d'une anaphore nominale du référent-thème dans la dernière acception référentielle qu'il avait eu en mémoire.

45. Discours Indirect Libre.

46. Voir règles valentiello-référentielles et syntactico-valentielle énoncées en introduction de cet article et Dupuy-Parant, 2006, 2007, 2008, 2010, 2012.

47. QJM, 3ème joie, p. 19, 1. 43 ; Mém., livre 3, chap. 5, p. 236, 1. 9-32 ; Mém., livre 3, chap. 5, p. 239, l. 9-11 (hors livres 4-5-6 des Mém.). Forme pronominale liée à la mémoire intra ou interséquentielle.

48. Chro., livre 1, chap. 247, p. 815, 1. 153. Le référent-thème apparaît en première mention sous la forme du pronom interrogatif que : si leur demanda que il lor falloit.

49. Chro., livre 1, chap. 253, p. 838, 1. 101-105; justifiable par la mémoire inter-séquentielle.

50. Ou en proposition infinitive.

51. Toute proposition peut être délimitée. Nous considérons qu'elles présentent une frontière d'entrée (début) et de sortie (fin). Ces frontières sont déterminées en fonction du nombre d'actants et de circonstants fonctionnant avec le verbe de la proposition : c'est le cas pour les frontières d'entrée et de sortie des propositions non-régies ainsi que pour la frontière de sortie des propositions régies qui ne sont pas spécifiquement marquées par des éléments ayant un rôle syntaxique délimitant. Pour la frontière d'entrée des propositions régies, elle présente le plus souvent un élément qui ouvre la proposition (conjonction, pronom ou adverbe-conjonctif) : cet élément détermine la frontière d'entrée en permettant l'enchâssement.

52. Dupuy, $2012: 298-299$.

53. Dupuy, 2015 et Dupuy-Parant, $2006: 527-553$.

54. Au préalable, contrairement aux passages de DI "seul" - sans lien avec du DD -, ceux où le DI est lié au DD sont quasiment absents des Chro. Cette étude a donc été réalisée essentiellement à partir des QJM et les Mém. (livre 4-5-6 des Mém. inclus).

55. Autres références : Mém., livre 4, chap. 5, p. 293, 1. 30-31; Mém. livre 4, chap. 11, p. 323, 1. 6-15. 56. QJM, $9^{\text {ème }}$ joie, p. 76, 1. 132-146 ; Mém., livre 4, chap. 3, p. 287, 1. 25-31.

57. Autre référence : Mém., livre 6, chap. 1, p. 424, 1. 26-33: récit: ces deux mil escus en or > cest argent / DI : les avoit receuz [...] les eust ambléz / DD : ce don (redéfinition : cause situationnelle) > le preigne [...] vous le mectréz icy / récit : $\underline{\text { son argent. }}$

58. Au sens de passeur de frontières.

59. Dans (17), il serait inexact de dire que le DI sert de passé référentiel au DD étant donné que les données référentielles ne sont pas introduites par anaphore nominale en DI mais en récit.

60. Une seule occurrence relevée dans le corpus.

61. Une hypothèse un peu hardie serait de considérer les pleurs de l'enfant comme une prise de parole qui servirait de préambule référentiel au DD.

62. Deuxième extrait : QJM, Tierce joye, p. 21-22, 1.124 à 135 (élargir le contexte aux lignes 96 à 135).

63. Corpus étendu à toutes les Joyes de mariage.

64. Autres références: Mém., livre 5, chap. 13, p. 379-280, 1. 22-2: DD : " je m'actends estre gouverneur de Flandres et m'y faire tout d'or " / DI anaphore conceptuelle avec changement de locuteur : « en $»$. 
65. Autre occurrence : QJM, Seconde joye, p. 15, 1. 40-53: «y a ung pou de jaleusie ».

66. Les articles signés Dupuy-Parant et Dupuy sont du même auteur.

\section{RÉSUMÉS}

Cet article, portant sur le moyen français, a pour objectif de pointer certains fonctionnements spécifiques à la continuité référentielle en discours direct et indirect à travers le choix des expressions anaphoriques qui se relaient au sein des chaînes anaphoriques (coréférence et anaphore associées). De ce point de vue, les occurrences relevées montreront un traitement différent des référents-thèmes et des référents-locuteurs et préciseront le traitement et le rôle de ces derniers quant au choix des expressions anaphoriques. Il sera également observé, à travers ces expressions anaphoriques, l'existence d'une mémoire intra et extra séquentielle ainsi que la particularité du discours indirect (voire du discours indirect libre) à combiner des spécificités du discours direct et avec celles du récit, servant ainsi de creuset ou de passeur référentiel entre le récit et/ou le discours direct.

This paper, concerning middle French, seeks to show some specifics mechanism of referential continuity in direct and indirect speech through the choice of the anaphoric expressions forming in chains (co-reference and anaphora associated). From this point of view, the data examined here reveal a different processing between referent-topic and referent-speakers and precise its specific processing and its role as for the choice of anaphoric expressions. Moreover, it will be observed through these anaphoric expressions, that an intra-sequential and extra-sequential memory and, furthermore, the characteristic of indirect speech (and potentially free indirect speech) which can combine direct speech characteristics with the narrative's ones. Thus, it's possible to call it a referential crucible or frontier runner between narrative and/or direct speech.

\section{INDEX}

Mots-clés : Continuité référentielle, discours direct et indirect, passerelles référentielles interdiscursives, chaînes anaphoriques, mémoire intra et extra-séquentielle en discours.

Keywords : Referential continuity, direct and indirect speech and narrative, referential interdiscursive link, anaphoric chains, intra-sequential and extra-sequential memory in direct or indirect speek.

\section{AUTEUR}

\section{ESTÈLE DUPUY}

Université de Poitiers, FoReLL A-EA 3816 